\title{
Percepção do ruído e seus efeitos em funcionários de um hospital universitário
}

\author{
Perception and effects of noise in employees of a university hospital \\ Percepción y efectos del ruido en empleados de un hospital universitario
}

Recebido: 21/01/2022 | Revisado: 29/01/2022 | Aceito: 02/02/2022 | Publicado: 04/02/2022

\author{
Priscila Silva Passos \\ ORCID: https://orcid.org/0000-0002-0520-899X \\ Pontifícia Universidade católica de São Paulo, Brasil \\ E-mail: priscilafga@gmail.com \\ Ana Claudia Fiorini \\ ORCID: https://orcid.org/0000-0003-2989-2308 \\ Pontifícia Universidade Católica de São Paulo, Brasil \\ E-mail: acfiorini@pucsp.br
}

\begin{abstract}
Resumo
Introdução: Funcionários de hospitais são expostos ao ruído proveniente de diversas fontes. Objetivo: Investigar a percepção e os efeitos do ruído em funcionários de um hospital universitário no município de Lagarto-SE. Método: Estudo observacional de corte transversal realizado com 170 funcionários. Foram aplicados anamnese e questionário de incômodo ao ruído, adaptado para o ambiente hospitalar. Níveis de ruído foram medidos nos turnos diurno e noturno. Foram encontrados valores de Leq entre 66,1 e $79 \mathrm{~dB}(\mathrm{~A})$, com níveis mais elevados no turno diurno. Para análise dos dados foi realizada aplicação de testes qui-quadrado de independência e ajustes de modelos de regressão logística. Resultados: A maioria dos profissionais era do sexo feminino $(69,4 \%)$, com idade média de 38 anos. A maioria $(54,1 \%)$ considerou o local repetidamente ou sempre ruidoso e $85,9 \%$ classificaram a intensidade moderada ou intensa. O período da manhã foi considerado o mais ruidoso. A fonte de ruído mais citada foram os profissionais. A maioria $(97,1 \%)$ acredita que o ruído no hospital pode prejudicar o paciente e $79,4 \%$ acredita que estratégias podem minimizar esta exposição, sendo a mais sugerida a realização de ações de conscientização aos profissionais (63\%). Conclusão: Houve percepção quando a frequência, a intensidade e a queixas relacionadas ao ruído, por parte dos profissionais.
\end{abstract}

Palavras-chave: Ruído; Saúde do trabalhador; Pessoal de saúde.

\begin{abstract}
Introduction: Hospital employees are exposed to noise from different sources. Objective: To investigate the perception and effects of noise in employees of a university hospital in the city of Lagarto-SE. Methods: Cross-sectional observational study. The sample consisted of 170 employees. The procedures included the application of anamnesis and a noise annoyance questionnaire adapted for the hospital environment. Noise levels were measured during day and night shifts. Leq values between 66.1 and $79 \mathrm{~dB}(\mathrm{~A})$ were found, with higher levels in the day shift. Data analysis was performed through the application of chi-square tests of independence and adjusted logistic regression models. Results: Most of the professionals interviewed were female $(69.4 \%)$, the mean age was 38 years old. Most professionals $(54.1 \%)$ considered the place repeatedly or always noisy and $85.9 \%$ classified its intensity as moderate or intense. The part of the day considered the noisiest was the morning. The main sources of noise cited by the research were hospital professionals. The majority $(97.1 \%)$ of the professionals reported that the noise in the hospital can harm the patient and $79.4 \%$ pointed out that strategies can be carried out to minimize such exposure. The measure most pointed out was the performance of awareness actions by professionals (63\%). Conclusion: We highlight the frequency, intensity, and complaints related to noise, on the part of the professionals.
\end{abstract}

Keywords: Noise; Occupational health; Health personnel.

\section{Resumen}

Introducción: Los empleados del hospital están expuestos al ruido de diferentes fuentes. Objetivo: Investigar la percepción y efectos del ruido en empleados de un hospital universitario de la ciudad de Lagarto-SE. Métodos: Estudio observacional transversal. La muestra estuvo conformada por 170 empleados. Los procedimientos incluyeron la aplicación de una anamnesis y un cuestionario de molestia por ruido adaptado al medio hospitalario. Los niveles de ruido se midieron durante los turnos de día y de noche. Se encontraron valores de Leq entre 66,1 y $79 \mathrm{~dB}(\mathrm{~A})$, con niveles más altos en el turno diurno. El análisis de los datos se realizó mediante la aplicación de pruebas de independencia chi-cuadrado y modelos de regresión logística ajustados. Resultados: La mayoría de los profesionales entrevistados eran del sexo femenino $(69,4 \%)$, la edad media fue de 38 años. La mayoría de los profesionales $(54,1 \%)$ consideró el lugar reiteradamente o siempre ruidoso y el 85,9\% clasificó su intensidad como moderada o intensa. La parte del día considerada más ruidosa fue la mañana. Las principales fuentes fueron los profesionales del hospital. La 
mayoría $(97,1 \%)$ relató que el ruido en el hospital puede perjudicar al paciente y el 79,4\% señaló que se pueden realizar estrategias para minimizar esa exposición. La más destacada fue la realización de acciones de sensibilización por parte de los profesionales (63\%). Conclusión: Destacamos la frecuencia, intensidad y quejas relacionadas con el ruido, por parte de los profesionales.

Palabras clave: Ruido; Salud laboral; Personal de salud.

\section{Introdução}

O ruído é considerado um agente poluidor cuja exposição tem aumentado com o crescimento e desenvolvimento das cidades, acompanhando os indivíduos em vários contextos, inclusive em seus ambientes de trabalho, sendo um dos riscos ocupacionais mais comuns em todo o mundo (Themann \& Masterson, 2019).

Pesquisas realizadas com diversas categorias profissionais estudaram o ruído ocupacional e seus efeitos na vida de trabalhadores como motoristas de caminhão (Lopes et al., 2007), professores (Fiorini \& Matos, 2009), trabalhadores da indústria têxtil (Alves \& Fiorini, 2012), pilotos civis (Falcão et al., 2014), trabalhadores de unidades de suporte móveis (Oliveira, Santos, Rabelo \& Magalhães, 2015), policiais militares (Gelardi \& Fiorini, 2016), músicos (Passos \& Fiorini, 2016; Muniz et al., 2017), trabalhadores industriais (Lin et al, 2018), dentre outros. Além destes, profissionais de saúde como enfermeiros e médicos têm sido expostos ao ruído em seu ambiente laboral (Ferreira, 2013; Ryan et al, 2016; Mcneer et al., 2017; Disher et al, 2017; Ornelas-Aguirre et al., 2018).

Apesar de serem considerados ambientes silenciosos, os hospitais podem oferecer riscos advindos da exposição sistemática ao ruído. Muitos deles localizam-se em grandes áreas urbanas, com ocorrência de poluição sonora. Além disso, pacientes e profissionais que trabalham neste ambiente são expostos ao ruído de conversas, sirenes, equipamentos e alarmes. Desta forma, um ambiente que deveria ser de tranquilidade para os que nele se encontram, torna-se desagradável, favorecendo sentimentos como estresse e ansiedade (Pereira et al., 3003).

O ruído no ambiente hospitalar pode gerar impactos em diversos aspectos relacionados à audição, emoção, fisiologia $\mathrm{e}$ à performance no trabalho. Para os funcionários do hospital, o ruído pode causar fadiga, irritabilidade, sintomas auditivos e interferências no desempenho que podem gerar riscos de acidentes de trabalho. Para os pacientes, pode causar estresse, aumento da pressão arterial sistólica, favorecendo o aumento no tempo de internação (Daraiseh et al., 2016).

Pesquisas realizadas indicam que os níveis de pressão sonora de hospitais em todo o mundo ultrapassam os limites estabelecidos pelas diretrizes da Organização Mundial da Saúde. Os dados coletados em vários hospitais indicam uma tendência no aumento dos níveis de ruído durante o dia e à noite, e muitas unidades exibem pouca ou nenhuma redução dos níveis sonoros durante a noite. As implicações desses resultados são significativas para pacientes, visitantes e funcionários do hospital (Busch-Vishniac et al, 2005).

Estudos que verifiquem os níveis de ruído no hospital e os efeitos trazidos por ele são relevantes por favorecerem o planejamento de ações que minimizem os efeitos deletérios do ruído neste local. Nesse sentido, o objetivo desse trabalho foi investigar a percepção e os efeitos do ruído em funcionários de um hospital universitário no município de Lagarto-SE.

\section{Metodologia}

Trata-se de um estudo observacional de corte transversal. O projeto foi submetido ao Comitê de Ética em Pesquisa da Pontifícia Universidade Católica de São Paulo, sendo aprovado com parecer de número 4.850.399. A pesquisa foi explicada verbalmente e um termo de consentimento livre e esclarecido (TCLE) foi entregue antes do início da coleta de dados.

A pesquisa foi realizada em um hospital universitário localizado no município de Lagarto-SE. A amostra foi composta por profissionais que atuam na instituição hospitalar, nos seguintes setores: Clínica médica, Eixo crítico (alas vermelha e amarela para adultos) e Unidade de terapia intensiva (UTI). A escolha das alas se deu devido aos diferentes perfis que estes 
locais apresentam entre si. Como critério de inclusão, os profissionais deveriam trabalhar há pelo menos seis meses na instituição, na assistência direta ao paciente, permanecendo toda a sua jornada de trabalho em um único setor.

Foram convidados a participar da pesquisa todos os médicos, fisioterapeutas, enfermeiros, técnicos de enfermagem e terapeutas ocupacionais que atuam, por toda a sua jornada de trabalho, nos setores supracitados. A seleção dos sujeitos foi feita com base nas escalas de trabalho dos profissionais, referentes ao mês de agosto de 2021. Os documentos contavam com uma lista de 220 profissionais, porém, foram excluídos aqueles afastados por motivos de doença, licenças, férias e aqueles que, por motivos de força maior, estavam afastados dos seus setores de trabalho no período da coleta de dados. No total, 170 profissionais se enquadraram nos critérios de inclusão e concordaram em responder os questionários.

Os profissionais foram abordados durante sua jornada de trabalho e convidados a participar da pesquisa, tanto no período diurno quanto no período noturno. Após a explicação dos procedimentos e aplicação do TCLE, os mesmos responderam à anamnese (que buscava informações sobre estado geral de saúde, histórico e queixas auditivas) e ao questionário de incômodo relacionado ao ruído, elaborado inicialmente por Juang et al. (2010), publicado na língua inglesa, e adaptado para o português por Ferreira (2013). O instrumento aborda questões relativas ao incômodo relacionado à exposição ao ruído no hospital e questões relativas aos efeitos que este ocasiona na saúde dos profissionais expostos. O instrumento é composto por sete seções relacionadas aos diversos efeitos advindos da exposição ao ruído, incluindo questões abertas e fechadas. Para as questões fechadas, as respostas variavam entre "nada, muito pouco, mais ou menos, bastante e extremamente". Os questionários foram aplicados presencialmente e lidos pela pesquisadora, que registrou as respostas em formulário digital via Microsoft Forms, em um tablet modelo S6 lite da marca Samsung.

Foram realizadas dosimetrias de ruído nos funcionários durante suas jornadas de trabalho, tanto no período diurno quanto no noturno, nas três alas selecionadas para a pesquisa. Para tanto, foi utilizado dosímetro, modelo Wed007, marca $01 \mathrm{~dB}$, devidamente calibrado antes de cada medição. As medições tiveram o objetivo de caracterizar a exposição ao ruído no ambiente de trabalho. Nesta etapa da pesquisa foram selecionados apenas técnicos de enfermagem, escolhidos aleatoriamente. Apenas um funcionário de cada setor em cada turno participou desta etapa.

$\mathrm{O}$ microfone foi posicionado próximo à orelha do funcionário, com o auxílio do cabo extensor. O aparelho mediu a dose individual do nível de pressão sonora em função do tempo, sendo configurado segundo os seguintes parâmetros (Fundacentro, 2001):

- $\quad$ Limite (dB): 85

- Taxa de troca $(\mathrm{dB}): 3$

- Limite Superior(dB): 115

- Nível do critério (dB): 85

- Resposta: Lenta

- Ponderação RMS: A

- Ponderação no pico: Z

As medições foram realizadas minuto a minuto, sendo obtidos os seguintes níveis:

- Leq: nível equivalente.

- Lmax: nível máximo.

- Lmin: nível mínimo.

- L10: nível sonoro que foi excedido em $10 \%$ do tempo de medição.

- L50: nível sonoro que foi excedido em 50\% do tempo de medição.

- L90: nível sonoro que foi excedido em 90\% do tempo de medição. 
Os dados das medições foram transferidos para o computador da pesquisadora, por meio do software dBWED e os valores calculados foram extraídos do software dBTrait.

A Tabela 1 apresenta os resultados das medições, de acordo com os parâmetros previamente definidos.

Tabela 1: Níveis de ruído encontrados nos setores investigados, por meio das dosimetrias, em $\mathrm{dB}(\mathrm{A})$.

\begin{tabular}{ccccccc}
\hline $\begin{array}{c}\text { SETORES POR } \\
\text { TURNO }\end{array}$ & Leq & Lmin & Lmax & L10 & L50 & L90 \\
\hline $\begin{array}{c}\text { Eixo Crítico } \\
\text { Diurno }\end{array}$ & 77,4 & 38,1 & 96,8 & 80,5 & 68,1 & 40,5 \\
\hline $\begin{array}{c}\text { Eixo Crítico } \\
\text { Noturno }\end{array}$ & 75,0 & 38,4 & 91,2 & 78,2 & 64,5 & 42,0 \\
\hline $\begin{array}{c}\text { Clínica Médica } \\
\text { Diurno }\end{array}$ & 77,9 & 49,1 & 92,7 & 81,2 & 69,5 & 58,6 \\
\hline $\begin{array}{c}\text { Clínica Médica } \\
\text { Noturno }\end{array}$ & 68,2 & 44,2 & 87,2 & 69,5 & 56,7 & 48,9 \\
\hline $\begin{array}{c}\text { UTI } \\
\text { Diurno }\end{array}$ & 79,8 & 42,1 & 105,9 & 78,0 & 63,5 & 43,5 \\
$\quad$ UTI & & & & & \\
Noturno & 66,1 & 44,0 & 87,0 & 67,7 & 53,9 & 46,6 \\
\hline
\end{tabular}

Leq= Nível equivalente, Lmin=nível mínimo, Lmax= nível máximo, L10= nível que indica que há $10 \%$ de valores inferiores (percentil 10), L50= mediana (percentil 50), L90= nível que indica que há $90 \%$ de valores inferiores (percentil 90). Fonte: Autores.

\section{Resultados}

Participaram da pesquisa 170 profissionais sendo $118(69,4 \%)$ do sexo feminino e $52(30,6 \%)$ no sexo masculino, com idades entre 24 e 73 anos. A média da idade foi de 38, mediana de 37 e desvio padrão de 7,69 anos. O tempo médio de profissão foi igual a 139 meses, desvio padrão de 73,13 e mediano de 132, sendo o tempo mínimo de 12 e máximo de 360 meses. Com relação ao tempo de trabalho na instituição, o tempo mínimo foi de seis meses, o tempo médio foi de 21,7 , desvio padrão igual a 16,37 e a mediana foi de 24 meses.

Foram entrevistados 37 (21,8\%) enfermeiros, 29 (17,1\%) fisioterapeutas, 13 (7,6\%) médicos, 88 (51,7\%) técnicos de enfermagem e três $(1,8 \%)$ terapeutas ocupacionais. Dentre os profissionais, a maioria deles trabalha no turno diurno (60,6\%), enquanto $34,7 \%$ no turno noturno e apenas oito (4,75\%) fazem um plantão de 24 horas. Com relação aos setores do hospital, a maioria deles atua no eixo crítico $(n=68-40 \%)$, seguidos dos que trabalham na clínica médica $(n=64-37 \%)$ e Unidade de Terapia Intensiva $(n=38-22,3 \%)$.

A maioria dos profissionais entrevistados relatou não se expor ao ruído em ambientes de lazer, incluindo uso de dispositivos de música pessoais $(\mathrm{n}=97-57,1 \%$ ). Com relação à queixa de dificuldade de entender a fala, a maioria $(\mathrm{n}=117-$ $68,8 \%$ ) afirmou não tê-la.

Com relação aos sintomas auditivos prévios, os resultados indicaram: incômodo a sons intensos $(n=74-43,5 \%)$, cefaleia $(n=74-43,5 \%)$, otalgia $(n=61-35,9 \%)$, zumbido $(n=59-34,7 \%)$, plenitude auricular $(n=51-30,0 \%)$, dor cervical $(n=38-22,3 \%)$, tontura $(n=36-21,2 \%)$ e otorreia $(n=16-9,4 \%)$.

Quando questionados sobre a frequência em que o seu ambiente de trabalho é ruidoso, 54,1\% relataram que o consideram "repetidamente" ou "sempre" ruidoso, 62 (36,5\%) "às vezes" ruidoso e apenas $16(9,4 \%)$ o consideraram "nunca" 
ou "raramente" ruidoso. Com relação ao nível do ruído, mais da metade dos indivíduos afirmaram considera-lo "moderado" (n=103, 60,6\%), 43 (25,3\%) “intenso", enquanto apenas 21 (12,3\%) "leve" ou “ausente”, e três relataram não saber.

Aos profissionais que trabalhavam do turno diurno e aos que davam plantões de 24 horas foi questionado o período do dia em que o ruído é pior. O período da manhã foi citado por 72,1\% desses profissionais, $10,8 \%$ à tarde e $1,8 \%$ o período da noite, enquanto 15,3\% afirmaram não saber. Quando questionados se eles acreditavam contribuir para o ruído existente, 49,4\% dos entrevistados responderam que sim, 4,1\% não souberam responder e os demais responderam que não.

Foi perguntado de forma aberta quais seriam as principais fontes de ruído do hospital. Por meio da Tabela 2, observase que as fontes mais relatadas foram: profissionais $(61,8 \%)$, bombas de infusão $(44,7 \%)$ e monitores $(43,5 \%)$.

Tabela 2. Distribuição de frequências da variável "Quais as principais fontes de ruído de dentro do hospital?”.

\begin{tabular}{lcc}
\hline \multicolumn{1}{c}{ Fonte de ruído } & $\mathrm{n} \%$ \\
\hline Profissionais & 105 & 61,8 \\
Manutenção/obras & 39 & 22,9 \\
Monitores & 74 & 43,5 \\
Bombas & 76 & 44,7 \\
Ventiladores & 37 & 21,8 \\
Portas & 17 & 10,0 \\
Lixeiras & 18 & 10,6 \\
Carrinhos/macas & 14 & 8,2 \\
Usuários & 49 & 28,8 \\
\hline
\end{tabular}

Fonte: Autores.

A Tabela 3 mostra que 50,6\%, 43,6\% e 59,4\% dos profissionais escolheram entre as categorias de resposta "Mais ou menos" ou "Bastante" para as variáveis "O ruido do ambiente te deixa estressado?", "O ruído do ambiente te causa falta de atenção?" e "O ruído do ambiente te incomoda?", respectivamente.

Tabela 3. Distribuição de frequências para a "Percepção subjetiva do ruído do ambiente” (n=170).

\begin{tabular}{c|cc|cc|ccc|cc|c}
\hline \multirow{2}{*}{ O ruído do ambiente te } & \multicolumn{2}{|c|}{ Nada } & \multicolumn{2}{c|}{ Muito pouco } & \multicolumn{2}{c|}{ Mais ou menos } & \multicolumn{2}{c|}{ Bastante } & \multicolumn{2}{c}{ Extremamente } \\
\cline { 2 - 12 } & $\mathrm{n}$ & $\%$ & $\mathrm{n}$ & $\%$ & $\mathrm{n}$ & $\%$ & $\mathrm{n}$ & $\%$ & $\mathrm{n}$ & $\%$ \\
\hline Deixa estressado & 50 & 29,4 & 29 & 17,1 & 53 & 31,2 & 33 & 19,4 & 5 & 2,9 \\
Causa falta de atenção & 58 & 34,1 & 32 & 18,8 & 36 & 21,2 & 38 & 22,4 & 6 & 3,5 \\
Incomoda & 30 & 17,7 & 31 & 18,2 & 48 & 28,2 & 53 & 31,2 & 8 & 4,7 \\
Causa tontura & 159 & 93,5 & 4 & 2,4 & 6 & 3,5 & 0 & 0,0 & 1 & 0,6 \\
\hline
\end{tabular}

Fonte: Autores.

Por meio da Tabela 4, nota-se que a maioria dos profissionais, 92,4\% e 88,8\%, escolheram a categoria "Nada" para as variáveis e "O ruído do ambiente provoca formigamento na cabeça?" e "O ruído do ambiente aumenta sua pressão?”, respectivamente. Por outro lado, observa-se que 53,6\% escolheram as categorias "Mais ou menos" ou "Bastante" para a variável "O ruído do ambiente dificulta a comunicação com os outros?”. 
Tabela 4. Distribuição de frequências para os "Efeitos do ruído na emoção" (n=170).

\begin{tabular}{c|cc|cc|cc|cc|cc}
\hline \multirow{2}{*}{ O ruído do ambiente } & \multicolumn{2}{|c|}{ Nada } & \multicolumn{2}{|c|}{ Muito pouco } & \multicolumn{2}{c|}{ Mais ou menos } & \multicolumn{2}{c|}{ Bastante } & \multicolumn{2}{c}{ Extremamente } \\
\cline { 2 - 12 } & $\mathrm{n}$ & $\%$ & $\mathrm{n}$ & $\%$ & $\mathrm{n}$ & $\%$ & $\mathrm{n}$ & $\%$ & $\mathrm{n}$ & $\%$ \\
\hline Dificulta sua comunicação com os outros & 43 & 25,3 & 28 & 16,5 & 47 & 27,7 & 44 & 25,9 & 8 & 4,7 \\
Provoca formigamento na cabeça & 157 & 92,4 & 6 & 3,5 & 6 & 3,5 & 1 & 0,6 & 0 & 0,0 \\
Aumenta sua pressão & 151 & 88,8 & 8 & 4,7 & 9 & 5,3 & 2 & 1,2 & 0 & 0,0 \\
Causa mau humor & 82 & 48,2 & 24 & 14,1 & 42 & 24,7 & 18 & 10,6 & 4 & 2,4 \\
Irrita & 53 & 31,2 & 28 & 16,5 & 61 & 35,9 & 22 & 12,9 & 6 & 3,5 \\
\hline
\end{tabular}

Fonte: Autores.

A análise da Tabela 5 indica que dentre a "Experiência do ruido de diferentes fontes nos quartos", as fontes que incomodam com mais frequência (Mais ou menos/Bastante/Extremamente) foram: Alarmes de monitoramento, pacientes gemendo ou chorando e equipamentos médicos.

Tabela 5. Distribuição de frequências da "Experiência do ruído de diferentes fontes nos quartos" ( $\mathrm{n}=170)$.

\begin{tabular}{c|cc|cc|cc|cc|cc}
\hline \multirow{2}{*}{ O ruído te incomoda? } & \multicolumn{2}{|c|}{ Nada } & \multicolumn{2}{|c|}{ Muito pouco } & \multicolumn{2}{c|}{ Mais ou menos } & \multicolumn{2}{c|}{ Bastante } & \multicolumn{2}{|c}{ Extremamente } \\
\cline { 2 - 12 } & $\mathrm{n}$ & $\%$ & $\mathrm{n}$ & $\%$ & $\mathrm{n}$ & $\%$ & $\mathrm{n}$ & $\%$ & $\mathrm{n}$ & $\%$ \\
\hline Abertura de gavetas ou armários & 110 & 64,7 & 30 & 17,7 & 17 & 10,0 & 8 & 4,7 & 5 & 2,9 \\
Aparelhos de sucção & 107 & 62,9 & 34 & 20,0 & 20 & 11,8 & 6 & 3,5 & 3 & 1,8 \\
Abertura e fechamento de portas & 74 & 43,5 & 32 & 18,8 & 33 & 19,4 & 21 & 12,4 & 10 & 5,9 \\
campainha & 116 & 68,3 & 14 & 8,2 & 16 & 9,4 & 19 & 11,2 & 5 & 2,9 \\
Alarmes de monitoramento & 49 & 28,8 & 25 & 14,7 & 39 & 22,9 & 46 & 27,1 & 11 & 6,5 \\
Pacientes gemendo ou chorando & 67 & 39,4 & 19 & 11,2 & 34 & 20,0 & 40 & 23,5 & 10 & 5,9 \\
Equipamentos médicos & 50 & 29,4 & 29 & 17,0 & 36 & 21,2 & 44 & 25,9 & 11 & 6,5 \\
Conversa de visitantes/ acompanhantes & 93 & 54,7 & 35 & 20,6 & 30 & 17,6 & 10 & 5,9 & 2 & 1,2 \\
\hline
\end{tabular}

Fonte: Autores.

A análise da Tabela 6 indica que dentre a "Experiência do ruído de diferentes fontes fora dos quartos", as fontes que incomodam com mais frequência (Mais ou menos/Bastante/Extremamente) foram: Profissionais falando alto ou gritando, carrinhos e macas, conversa entre profissionais e Telefones tocando.

Tabela 6. Distribuição de frequências da "Experiência do ruido de diferentes fontes de fora do quarto" (n=170).

\begin{tabular}{|c|c|c|c|c|c|c|c|c|c|c|}
\hline \multirow{2}{*}{ O ruído te incomoda? } & \multicolumn{2}{|c|}{ Nada } & \multicolumn{2}{|c|}{ Muito pouco } & \multicolumn{2}{|c|}{ Mais ou menos } & \multicolumn{2}{|c|}{ Bastante } & \multicolumn{2}{|c|}{ Extremamente } \\
\hline & $\mathrm{n}$ & $\%$ & $\mathrm{n}$ & $\%$ & $\mathrm{n}$ & $\%$ & $\mathrm{n}$ & $\%$ & $\mathrm{n}$ & $\%$ \\
\hline Impressora & 155 & 91,2 & 14 & 8,2 & 1 & 0,6 & 0 & 0,0 & 0 & 0,0 \\
\hline Troca de turno dos profissionais & 75 & 44,1 & 34 & 20,0 & 25 & 14,7 & 30 & 17,7 & 6 & 3,5 \\
\hline Profissionais falando alto ou gritando & 34 & 20,0 & 24 & 14,1 & 45 & 26,5 & 56 & 32,9 & 11 & 6,5 \\
\hline Carrinhos/macas & 71 & 41,8 & 31 & 18,2 & 36 & 21,2 & 23 & 13,5 & 9 & 5,3 \\
\hline Auto falante & 135 & 79,4 & 11 & 6,5 & 9 & 5,3 & 13 & 7,6 & 2 & 1,2 \\
\hline Celulares & 98 & 57,6 & 30 & 17,6 & 29 & 17,1 & 10 & 5,9 & 3 & 1,8 \\
\hline Telefones tocando & 89 & 52,3 & 30 & 17,6 & 27 & 15,9 & 20 & 11,8 & 4 & 2,4 \\
\hline Passos (caminhar) & 139 & 81,7 & 17 & 10,0 & 11 & 6,5 & 2 & 1,2 & 1 & 0,6 \\
\hline Limpeza dos ambientes & 141 & 82,9 & 16 & 9,4 & 10 & 5,9 & 3 & 1,8 & 0 & 0,0 \\
\hline Conversa entre os profissionais & 78 & 45,9 & 38 & 22,3 & 29 & 17,1 & 21 & 12,3 & 4 & 2,4 \\
\hline $\begin{array}{c}\text { Conversa dos visitantes/ } \\
\text { acompanhantes }\end{array}$ & 116 & 68,2 & 26 & 15,3 & 20 & 11,8 & 7 & 4,1 & 1 & 0,6 \\
\hline
\end{tabular}

Fonte: Autores. 
A Tabela 7 avalia o "Impacto do ruído ambiente na performance do trabalho" e mostra que a resposta que apareceu com maior frequência (Mais ou menos/Bastante/Extremamente) foi "Entender o que os outros estão falando" (55,3\%).

Tabela 7. Distribuição de frequências da variável “Impacto do ruído ambiente na performance do trabalho" (n=170).

\begin{tabular}{c|cc|cc|cc|cc|cc|}
\hline \multirow{2}{*}{ O ruído atrapalha? } & \multicolumn{2}{|c|}{ Nada } & \multicolumn{2}{|c|}{ Muito pouco } & \multicolumn{2}{c|}{ Mais ou menos } & \multicolumn{2}{|c|}{ Bastante } & \multicolumn{2}{c}{ Extremamente } \\
\cline { 2 - 13 } & $\mathrm{n}$ & $\%$ & $\mathrm{n}$ & $\%$ & $\mathrm{n}$ & $\%$ & $\mathrm{n}$ & $\%$ & $\mathrm{n}$ & $\%$ \\
\hline Eficiência no trabalho & 73 & 43,0 & 38 & 22,3 & 38 & 22,3 & 19 & 11,2 & 2 & 1,2 \\
Raciocínio & 53 & 31,2 & 30 & 17,6 & 52 & 30,6 & 29 & 17,1 & 6 & 3,5 \\
Execução de tarefas & 69 & 40,6 & 38 & 22,3 & 32 & 18,8 & 28 & 16,5 & 3 & 1,8 \\
Entender o que os outros estão falando & 27 & 15,9 & 38 & 22,3 & 49 & 28,8 & 45 & 26,5 & 11 & 6,5 \\
Atenção & 65 & 38,2 & 30 & 17,7 & 39 & 22,9 & 30 & 17,7 & 6 & 3,5 \\
\hline
\end{tabular}

Fonte: Autores.

Os profissionais foram questionados se acreditavam que o ruído no ambiente hospitalar poderia prejudicar o pacientes. A grande maioria (97,1\%) afirmou que sim. Destes, $84,8 \%$ acham que o ruído no ambiente atrapalha o sono, $68,5 \%$ que causa irritabilidade, $66,7 \%$ que causa estresse, $49,7 \%$ alterações comportamentais, 15,2\% acreditam que afeta na recuperação clínica, 12,7\% que causa alterações auditivas e 4,2\% que interfere na assistência adequada ao paciente. Nesta pergunta os profissionais poderiam apontar mais uma consequência relacionada ao ruído.

Quando questionados se o ruído no hospital poderia prejudicar os profissionais, $88,8 \%$ afirmaram que sim. Entre esses, 70,2\% acham que o ruído causa estresse, 66,9\% acham que altera a atenção, 66,9\% que causa irritabilidade, 41,1\% acreditam que causa cansaço, 39,7\% que causa cefaleia, $21,2 \%$ que causa alterações auditivas.

Os entrevistados também foram questionados se o ruído hospitalar poderia prejudicar os acompanhantes ou visitantes dos pacientes internados. 100 (58,8\%) deles acreditam que sim. Destes, 67\% acredita que causa irritabilidade, 63\% que causa estresse, $27 \%$ que causa cansaço, $25 \%$ que altera a atenção, $19 \%$ afirmam que causa cefaleia, $10 \%$ que interfere na comunicação, $10 \%$ que interfere no sono e $5 \%$ acreditam que pode causar alterações auditivas.

Ao final do questionário foi perguntado aos entrevistados se algo poderia ser feito para melhorar o ruído no ambiente hospitalar. E caso fosse possível, quais seriam as suas sugestões. Apesar de 17 pessoas acreditarem que não é possível melhorar este aspecto e 18 relatarem não saber responder, a maioria $(n=135-79,4 \%)$ acredita que existem estratégias para minimizar esta exposição. Dentre estes, 63\% sugere ações de conscientização aos profissionais, $28,9 \%$ sugerem ajustes em equipamentos médicos, $23,7 \%$ sugerem alterações no espaço físico do hospital, $17 \%$ sugerem ações de conscientização aos usuários e 16,3\% alterações na logística.

\section{Discussão}

Ao analisar o perfil dos profissionais que compuseram a amostra, pode-se verificar que a maioria era do sexo feminino, com idade média de 38 anos. Esse perfil corrobora outros estudos realizados no ambiente hospitalar, e é compatível com o fato de que grande parte das categorias profissionais na área da saúde é composta principalmente por mulheres (Costa et al., 2013; Sampaio et al, 2010; Filus et al., 2018). A categoria profissional com maior número de indivíduos foi a dos técnicos de enfermagem, assim como no estudo de Sampaio e colaboradores (2010), realizado com a equipe multidisciplinar de um hospital.

A maioria dos profissionais que participou da pesquisa atuava no turno diurno, cuja jornada de trabalho tem início as 7:00 e é finalizada as 19:00 horas. A coleta foi baseada nas escalas de trabalho de cada setor, que apresentavam o número 
maior de funcionários escalados para este turno. Além disso, durante o turno noturno, os profissionais tem um intervalo maior para descanso, o que diminuiu a disponibilidade em participar da pesquisa.

Os profissionais do turno Diurno permanecem no hospital durante os períodos da manhã, da tarde e início da noite, sendo assim, era esperado que apenas estes e os que têm jornada de trabalho de 24 horas pudessem responder qual o período do dia eles consideram mais ruidoso. Dentre os que responderam a essa pergunta, a grande maioria referiu o turno da manhã (72,1\%), diferente dos achados do estudo Filus et al (2018), realizado em um pronto socorro, cuja amostra considerou o período da tarde como mais ruidoso. As medições de ruído realizadas neste estudo tiveram o intuito de caracterizar o ambiente, mas acabaram confirmando as pesquisas supracitadas, uma vez que os níveis sonoros foram mais elevados durante o dia, em todas as alas avaliadas (Leq diurno de 77,4 a 79,8 dB(A) e Leq noturno de 66,1 a $75 \mathrm{~dB}(\mathrm{~A})$ ).

O nível do ruído foi percebido como moderado por $60,6 \%$ dos profissionais entrevistados, enquanto $25,3 \%$ consideraram como intenso. Tais resultados são semelhantes aos encontrados por Ferreira (2013), que estudou a exposição de profissionais de enfermagem ao ruído no ambiente hospitalar e identificou que 59\% dos indivíduos classificaram o ruído como moderado e $24,2 \%$ como intenso. Sampaio e colaboradores (2010) investigaram a percepção dos profissionais quanto ao ruído na UTI e 97,3\% deles classificaram como moderado a intenso.

A percepção de que o ruído é mais intenso era esperada devido aos níveis sonoros encontrados nas medições realizadas neste estudo. Os resultados estavam acima dos recomendados pelas normas vigentes para ambientes hospitalares em todas as alas pesquisadas, nos períodos diurno e noturno, com Leq entre 66,1 e 79 dB(A). A Organização Mundial de Saúde (OMS, 2000) recomenda para hospitais níveis equivalentes (Leq) de até $40 \mathrm{~dB}(\mathrm{~A})$ para o período diurno e de $35 \mathrm{~dB}(\mathrm{~A})$ para o período noturno; enquanto a Environmental Protection Agency recomenda que os níveis de ruído em hospitais não ultrapassem $45 \mathrm{~dB}(\mathrm{~A})$. Quanto à legislação trabalhista, os níveis não estão acima do máximo permitido para a NR 15 e que representa risco à audição ( $85 \mathrm{~dB}(\mathrm{~A})$ para jornada diária de oito horas). Entretanto, vale ressaltar que tal norma não tem aplicação direcionada a trabalhadores de hospitais, pois o que deve ser considerado não é exclusivamente o risco à audição, mas sim outras alterações à saúde e ao desempenho no trabalho. Assim, o adequado seria considerar a NR 17, uma vez que esta tem como objetivo estabelecer as diretrizes e os requisitos que permitam a adaptação das condições de trabalho às características psicofisiológicas dos trabalhadores, de modo a proporcionar conforto, segurança, saúde e desempenho eficiente no trabalho.

Dentre outros, o item da NR 17 intitulado "Condições de conforto no ambiente de trabalho" indica que para locais de trabalho em ambientes internos onde são executadas atividades que exijam solicitação intelectual e atenção constantes, devem ser adotadas medidas de conforto acústico e térmico. No caso do conforto acústico, o nível de ruído de fundo deve respeitar os valores de referências das normas técnicas oficiais. Nos demais casos, o nível de ruído de fundo aceitável para efeito de conforto acústico será de até $65 \mathrm{~dB}(\mathrm{~A})$ (Brasil, 1990).

No Brasil, a norma técnica oficial para conforto acústico é a NBR 10.152 da ABNT, cuja primeira publicação foi em 1997. Esta Norma Brasileira determina para ambientes hospitalares níveis sonoros aceitáveis (dB(A)) e níveis de conforto (NC) de acordo com os locais, a saber: entre 35 e $45 \mathrm{~dB}(\mathrm{~A})$ e $\mathrm{NC}$ entre 30 e $40 \mathrm{~dB}(\mathrm{~A})$ em apartamentos, enfermarias, berçários ou centros cirúrgicos; entre 40 e $50 \mathrm{~dB}(\mathrm{~A})$ e NC entre 35 e $45 \mathrm{~dB}(\mathrm{~A})$ para laboratórios e áreas para uso público e, finalmente, entre 45 a $55 \mathrm{~dB}(\mathrm{~A})$ e $\mathrm{NC}$ entre 40 e $50 \mathrm{~dB}(\mathrm{~A})$ para os setores de serviços hospitalares (ABNT, 1997; BRASIL, 1990). A partir destas informações, é notório que os níveis sonoros avaliados na presente pesquisa estão muito superiores aos níveis de conforto estabelecidos para ambientes hospitalares.

Outros estudos realizados em ambientes hospitalares também identificaram níveis de ruído que extrapolavam os limites recomendados e poderiam representar riscos à saúde dos trabalhadores (Pereira et al, 2003; Bu sch-Vishniac et al, 2005; Carvalho et al, 2005; Sampaio et al, 2010; Daraiseh et al, 2016; Costa et al, 2013; Oliveira et al, 2013; Ryan et al, 2016; Ornelas-Aguirre et al, 2018). 
Quando questionados sobre as principais fontes de ruído dentro do hospital, as mais citadas pelos profissionais foram as provenientes dos próprios funcionários $(61,8 \%)$, as bombas de infusão $(44,7 \%)$ e os monitores $(43,5 \%)$. Estas também foram as mais relatadas como causadoras de incômodo, dentro e fora dos quartos. Resultados semelhantes, que também identificaram conversas entre profissionais como importante fonte de ruído ambiental, foram obtidos em outras pesquisas realizadas em ambientes hospitalares (Ferreira, 2013; Oliveira et al, 2013; Daraiseh et al, 2016). Cabe ressaltar que a percepção quanto às fontes é subjetiva e pode variar de acordo com cada indivíduo e/ou categoria profissional. Em estudo realizado por Filus et al (2018), que comparou a percepção entre profissionais das áreas de enfermagem e administrativa frente ao ruído no pronto-socorro, os profissionais da enfermagem apresentaram maior percepção para o ruído dos equipamentos (60,53\%) enquanto os da área administrativa, para o ruído de pessoas $(85,71 \%)$.

Os efeitos do ruído percebidos de forma mais intensa pelos profissionais foram o incômodo, a dificuldade de comunicação, o estresse e a irritabilidade. Tais resultados corroboram outros estudos que identificaram elevada ocorrência de efeitos como estresse (Ryherd et al., 2008; Carvalho et al, 2005; Filus et al, 2018), irritabilidade (Costa et al, 2013; Filus et al, 2018) e incômodo (Costa et al, 2013; Andrade et al., 2016; Nazario et al, 2015); relatados por funcionários de hospitais.

Quanto ao impacto que o ruído pode causar na performance do trabalho, as principais queixas estavam relacionadas às interferências no raciocínio e na dificuldade de compreensão de fala. Nazário et al (2015) estudaram os efeitos do ruído na rotina de trabalho em uma unidade neonatal de um hospital universitário, e citaram a diminuição da concentração, irritabilidade, estresse, distração, agitação e alterações auditivas. Em estudo realizado por Ferreira (2013), houve associação entre o ambiente ruidoso e a percepção dos profissionais de que o ruído do hospital pode dificultar o entendimento de fala.

Quando questionados acerca da frequência, $54,1 \%$ dos profissionais classificaram o ambiente como repetidamente ruidoso e 36,5\% como às vezes ruidoso. As análises estatísticas realizadas por desfecho mostram que a ocorrência de algumas queixas aumentou à medida que o ruído do ambiente tornou-se mais frequente. Estes resultados se aplicaram para as queixas de cansaço, estresse, irritabilidade, mal humor, dificuldade de comunicação e o incômodo quanto aos equipamentos médicos, alarmes, telefones e ao ruído proveniente dos profissionais. Em estudo realizado por Ferreira (2013), foram encontradas associações significantes entre a percepção de que o ambiente é barulhento e os desfechos estresse, incômodo e cansaço.

Daraiseh et al (2016) examinaram a correlação entre níveis de pressão sonora (NPS), frequência cardíaca e estresse em enfermeiros de uma unidade de internação pediátrica. As análises indicaram que o ruído foi significativamente associado à frequência cardíaca, mas não ao estresse.

A queixa de incômodo a sons intensos, relatada na anamnese, esteve associada a algumas variáveis relacionadas ao ruído no ambiente de trabalho, como cansaço, dor de cabeça, mau humor, dificuldade de comunicação, falta de atenção, estresse e irritabilidade. Além disso, a proporção de profissionais que se incomodam com o ruído de alarmes, equipamentos médicos e o ruído proveniente dos profissionais foi maior entre aqueles que já tiveram ou têm incômodo a sons intensos.

A profissão foi uma variável que esteve associada a alguns desfechos da pesquisa. As análises evidenciaram que, quando comparados a profissionais de outras categorias, os técnicos de enfermagem relataram menos incômodo ao ruído de equipamentos médicos, de alarmes, de celulares, de telefones tocando e ao ruído proveniente de profissionais. Com relação ao ruído no ambiente de trabalho, essa categoria apresentou, ainda, menos queixas de estresse, incômodo e falta de atenção; além de menos queixas relacionadas à performance no trabalho, como comprometimento na eficiência e na execução de tarefas. Tal achado é relevante, uma vez que os técnicos de enfermagem são profissionais que atuam diretamente em contato com o paciente por maiores períodos de tempo, se comparados a outras categorias. Entretanto, durante as entrevistas, muitos relataram que se acostumaram com o ruído do ambiente, fato que pode ser explicado pela possibilidade da ocorrência de acomodação auditiva que ocorre, por exemplo, na fadiga de alarmes (Sykes et al, 2011). 
O setor de atuação profissional esteve associado aos seguintes desfechos: "O ruído do ambiente atrapalha o raciocínio", "O ruído do ambiente te atrapalha de entender o que os outros estão falando?", "O ruído do ambiente te deixa desatento?" e "O ruído de equipamentos médicos te incomoda?". A partir dos resultados, foi possível observar que os profissionais menos afetados foram aqueles que atuavam na clínica médica. Tal achado pode ser explicado pelo fato de os demais setores da pesquisa (UTI e Eixo Crítico) atendem pacientes com quadros clínicos mais graves, alguns em estágio agudo da doença, e consequentemente, demandam maior atenção e medidas imediatas por parte dos profissionais (Filus et al, 2014; Bolela e Jericó, 2006). Além disso, são setores que apresentam um número maior de equipamentos médicos que representam fontes de ruído intenso como, por exemplo, bombas de infusão, monitores e ventiladores mecânicos. A predominância desses equipamentos nessas alas pode justificar, inclusive, o fato de que a maioria das pesquisas que investigam o ruído em ambientes hospitalares é realizada em unidades de terapia intensiva (Carvalho et al, 2005; Ryherd et al, 2008; Macedo et al, 2009; Sampaio et al, 2010; Oliveira et al, 2013; Johansson et al., 2016; Knauert et al, 2016).

Apesar das informações supracitadas, as medições de ruído realizadas identificaram níveis sonoros muito semelhantes nas três alas (eixo crítico, UTI e clínica médica). No período diurno, o nível equivalente de ruído obtido na clínica médica foi maior que o encontrado no eixo crítico. Já no período noturno, os menores níveis foram obtidos na UTI. Vale ressaltar que tais medições foram realizadas apenas para caracterizar os ambientes de trabalho dos profissionais, mas foram fundamentais para indicar a importância de futura avaliação ambiental em todas as alas do hospital.

Os modelos de regressão logísticas ajustados identificaram resultados de grande importância para reconhecer o comportamento entre as variáveis desfechos e as explicativas, relacionadas aos dados obtidos com os profissionais que atuam no hospital. De forma sucinta e aplicada para todos os profissionais é possível afirmar que quanto maior a percepção do ruído no ambiente, maiores serão as seguintes queixas: incômodo, mau humor, cansaço, interferência na eficiência no trabalho, dor de cabeça, incômodo com ruídos de equipamentos, estresse, irritabilidade, nervosismo, alteração na produtividade e cansaço visual.

Entretanto, para algumas variáveis, foi possível observar que os dados diferem de acordo com a profissão. O enfermeiro percebe mais que o ruído pode dificultar a comunicação, quanto maior o tempo de trabalho. Enfermeiros e médicos percebem mais que o ruído atrapalha o raciocínio, a execução de tarefas, a compreensão de fala, aumenta o incômodo, provoca desatenção, altera a produtividade. Tais percepções estão relacionadas ao tempo de trabalho, ou seja, quanto maior o tempo, maior a percepção. Estas duas categorias também foram as que mais reclamaram de profissionais falando alto ou gritando e da conversa entre profissionais, principalmente aqueles que atuavam no Pronto Socorro e que tinham mais tempo de trabalho.

A maioria dos profissionais entrevistados acredita que o ruído pode prejudicar tanto os pacientes $(97,1 \%)$ quanto os visitantes $(58,8 \%)$ e os próprios funcionários $(88,8 \%)$. Além disso, $79,4 \%$ acreditam que existem estratégias que possam minimizar a exposição ao ruído no ambiente hospitalar. As principais medidas apontadas foram a conscientização dos profissionais e os ajustes em equipamentos. Ações voltadas para a conscientização de profissionais e usuários foram sugeridas em outros estudos como estratégias para reduzir o ruído em hospitais (Carvalho et al, 2005; Oliveira et al, 2013; Filus et al, 2014; Andrade et al, 2016; Daraiseh et al, 2016; Johansson et al, 2016; Ornelas-Aguirre et al, 2018; Joseph, Mehazabeen \& U, 2020). Estudos realizados em hospitais sugeriram outras estratégias de controle como mudanças estruturais no espaço físico (Johansson et al, 2016; Mcneer et al, 2017; Juang et al., 2020) e manutenção em equipamentos (Nazário et al, 2015; Andrade et al, 2016).

\section{Conclusão}

Houve percepção quando a frequência, a intensidade e a queixas relacionadas ao ruído, por parte dos profissionais. A maioria deles acredita que o ruído do hospital pode prejudicar os funcionários, os pacientes internados e seus visitantes. Além disso, acredita que estratégias possam minimizar o ruído no ambiente hospitalar. A conscientização dos profissionais e os 
ajustes em equipamentos foram as medidas mais sugeridas. A categoria profissional, a queixa de incômodo a sons intensos e a percepção quanto à frequência do ruído foram as variáveis mais associadas à percepção e ao incômodo ao ruído no ambiente hospitalar.

\section{Referências}

Alves, A. S., \& Fiorini, A. Claudia. (2012). A autopercepção do handicap auditivo em trabalhadores de uma indústria têxtil. Distúrb. Comun, 24(3), 337-349.

Andrade, K. P., Oliveira, L. L. A. de, Souza, R. de P., \& Matos, I. M. de. (2016). Medida do nível de ruído hospitalar e seus efeitos em funcionários a partir do relato de queixas. Revista CEFAC, 18(6), 1379-1388. https://doi.org/10.1590/1982-0216201618619815

Brasil. (1990). Portaria no 3.751, de 23 de novembro de 1990. Normas regulamentadoras de segurança e saúde no trabalho (NR-17): Ergonomia. Ministério do Trabalho e Emprego.

Busch-Vishniac, I. J., West, J. E., Barnhill, C., Hunter, T., Orellana, D., \& Chivukula, R. (2005). Noise levels in Johns Hopkins Hospital. The Journal of the Acoustical Society of America, 118(6), 3629-3645. https://doi.org/10.1121/1.2118327

Carvalho, W. B., Pedreira, M. L. G., \& Aguiar, M. A. L. de. (2005). Nível de ruídos em uma unidade de cuidados intensivos pediátricos. Jornal de Pediatria, 81(6), 495-498. https://doi.org/10.1590/S0021-75572005000800015

Costa, G. de L., Lacerda, A. B. M. de, \& Marques, J. (2013). Ruído no contexto hospitalar: impacto na saúde dos profissionais de enfermagem. Revista CEFAC, 15(3), 642-652. https://doi.org/10.1590/S1516-18462013005000012

Daraiseh, N. M., Hoying, C. L., Vidonish, W. P., Lin, L., \& Wagner, M. (2016). Noise Exposure on Pediatric Inpatient Units. JONA: The Journal of Nursing Administration, 46(9), 468-476. https://doi.org/10.1097/NNA.0000000000000377

Disher, T. C., Benoit, B., Inglis, D., Burgess, S. A., Ellsmere, B., Hewitt, B. E., Bishop, T. M., Sheppard, C. L., Jangaard, K. A., Morrison, G. C., \& CampbellYeo, M. L. (2017). Striving for Optimum Noise-Decreasing Strategies in Critical Care. Journal of Perinatal \& Neonatal Nursing, 31(1), 58-66. https://doi.org/10.1097/JPN.0000000000000229

Falcão, T. P., Luiz, R. R., Schütz, G. E., Mello, M. G. da S., \& Câmara, V. de M. (2014). Audiometric profile of civilian pilots according to noise exposure. Revista de Saúde Pública, 48(5), 790-796. https://doi.org/10.1590/S0034-8910.2014048005256

Ferreira, A. (2013). Percepção do ruído hospitalar em funcionários de uma maternidade do município de São Bernardo do Campo.

Filus, W. A., Sampaio, J. M. R., Albizu, E. J., Marques, J. M., \& Lacerda, A. B. M. de. (2018). Percepção de equipes de trabalho sobre o ruído em prontosocorro. Audiology - Communication Research, 23(0). https://doi.org/10.1590/2317-6431-2018-2014

Fiorini, A., \& Matos, E. (2009). Ruído na escola: queixas de saúde e o incômodo em professores do ensino público. Distúrb Comun, 21 (2), $187-197$.

Fundacentro. (2001). Norma de Higiene Ocupacional. Avaliação da exposição ocupacional ao ruído. https://www.areaseg.com/bib/10\%20$\% 20$ NHO\%20Normas\%20de\%20Higiene\%20Ocupacional/NHO-01.pdf >.

Gelardi, V. C., \& Fiorini, A. C. (2016). Efeitos auditivos do ruído e dificuldades de comunicação em um grupamento de radiopatrulha aérea. Disturb Comun, 28(4), 709-717.

Johansson, L., Knutsson, S., Bergbom, I., \& Lindahl, B. (2016). Noise in the ICU patient room - Staff knowledge and clinical improvements. Intensive and Critical Care Nursing, 35, 1-9. https://doi.org/10.1016/j.iccn.2016.02.005

Joseph, B. E., Mehazabeen, H., \& U, M. (n.d.) (2020). Noise pollution in hospitals - A study of public perception. Noise \& Health, 22(104), 28-33. https://doi.org/10.4103/nah.NAH_13_20

Juang, D., Lee, C., Yang, T., \& Chang, M. (2010). Noise pollution and its effects on medical care workers and patients in hospitals . Int. J. Environ. Sci. Tech, $7(4), 705-716$.

Jung, S., Kim, J., Lee, J., Rhee, C., Na, S., \& Yoon, J.-H. (2020). Assessment of Noise Exposure and Its Characteristics in the Intensive Care Unit of a Tertiary Hospital. International Journal of Environmental Research and Public Health, 17(13), 4670. https://doi.org/10.3390/ijerph17134670

Knauert, M., Jeon, S., Murphy, T. E., Yaggi, H. K., Pisani, M. A., \& Redeker, N. S. (2016). Comparing average levels and peak occurrence of overnight sound in the medical intensive care unit on A-weighted and C-weighted decibel scales. Journal of Critical Care, 36, 1-7. https://doi.org/10.1016/j.jcrc.2016.06.005

Lin, J., Wang, H., Yan, F., Tang, K., Zhu, H., Weng, Z., \& Wang, K. (2018). Effects of occupational exposure to noise and dust on blood pressure in Chinese industrial workers. Clinical and Experimental Hypertension, 40(3), 257-261. https://doi.org/10.1080/10641963.2017.1368534

Lopes, G., Russo, I. C. P., \& Fiorini, A. C. (2007). Estudo da audição e da qualidade de vida em motoristas de caminhão. Revista CEFAC, 9(4), 532-542. https://doi.org/10.1590/S1516-18462007000400014

McNeer, R. R., Bennett, C. L., Horn, D. B., \& Dudaryk, R. (2017). Factors Affecting Acoustics and Speech Intelligibility in the Operating Room. Anesthesia \& Analgesia, 124(6), 1978-1985. https://doi.org/10.1213/ANE.0000000000002118

Muniz, C. M. D. C., Amorim, C. M. T., Felipe, I. M. A., \& Dias, R. da S. (2018). Perfil audiométrico de músicos profissionais: revisão sistemática. Revista Brasileira Em Promoção Da Saúde, 31(1), 1-8. https://doi.org/10.5020/18061230.2018.6674 
Research, Society and Development, v. 11, n. 2, e51211225998, 2022

(CC BY 4.0) | ISSN 2525-3409 | DOI: http://dx.doi.org/10.33448/rsd-v11i2.25998

Nazario, A. P., Santos, V. C. B. J., Rossetto, E. G., Souza, S. N. D. H. de, Amorim, N. E. Z., \& Scochi, C. G. S. (2015). Avaliação dos ruídos em uma unidade neonatal de um hospital universitário. Semina: Ciências Biológicas e Da Saúde, 36(1Supl), 189. https://doi.org/10.5433/1679-0367.2015v36n1Suplp189

Oliveira, F. M. do C. da S. N. de, Paiva, M. B. de, Nascimento, M. A. de L., Rezende, V. M., Silva, A. S. da, \& Silva, C. R. L. da. (2013). Noise levels in a pediatric intensive care unit: an observational and correlational study. Online Brazilian Journal of Nursing, 12(3). https://doi.org/10.5935/16764285.20134043

Oliveira, R. C., Santos, J. N., Rabelo, A. T. V., \& Magalhães, M. de C. (2015). The impact of noise exposure on workers in Mobile Support Units. CoDAS, 27(3), 215-222. https://doi.org/10.1590/2317-1782/20152014136

Organização Mundial da Saúde (OMS). (2000). Guidelines for community noise. http://whqlibdoc.who.int/hq/1999/a68672.pdf

Ornelas-Aguirre, J. M., Zárate-Coronado, O., Gaxiola-González, F., \& Neyoy-Sombra, V. (2018). Nivel de ruido ambiental en 2 unidades de cuidados críticos de un centro de tercer nivel de atención. Archivos de Cardiología de México, 88(4), 253-260. https://doi.org/10.1016/j.acmx.2017.02.007

Passos, P. S., \& Fiorini, A. C. (2016). Efeitos auditivos em jovens músicos de uma banda filarmônica. Disturb Comun, 28(3).

Pereira, R. P., Toledo, R. N., Amaral, J. L. G. do, \& Guilherme, A. (2003). Qualificação e quantificação da exposição sonora ambiental em uma unidade de terapia intensiva geral. Revista Brasileira de Otorrinolaringologia, 69(6), 766-771. https://doi.org/10.1590/S0034-72992003000600007

Ryan, K. M., Gagnon, M., Hanna, T., Mello, B., Fofana, M., Ciottone, G., \& Molloy, M. (2016). Noise Pollution: Do We Need a Solution? An Analysis of Noise in a Cardiac Care Unit. Prehospital and Disaster Medicine, 31(4), 432-435. https://doi.org/10.1017/S1049023X16000388

Ryherd, E. E., Waye, K. P., \& Ljungkvist, L. (2008). Characterizing noise and perceived work environment in a neurological intensive care unit. The Journal of the Acoustical Society of America, 123(2), 747-756. https://doi.org/10.1121/1.2822661

Sampaio Neto, R. de A., Mesquita, F. O. de S., Paiva Junior, M. D. S., Ramos, F. F., Andrade, F. M. D. de, \& Correia Junior, M. A. de V. (2010). Ruídos na unidade de terapia intensiva: quantificação e percepção dos profissionais de saúde. Revista Brasileira de Terapia Intensiva, 22(4), 369-374. https://doi.org/10.1590/S0103-507X2010000400010

Themann, C. L., \& Masterson, E. A. (2019). Occupational noise exposure: A review of its effects, epidemiology, and impact with recommendations for reducing its burden. The Journal of the Acoustical Society of America, 146(5), 3879-3905. https://doi.org/10.1121/1.5134465.

United States Environmental Protection Agency. (1974). Information on levels of environmental noise requisite to protect public health and welfare with an adequate margin of safety. Government Printing Office. 